\title{
CLOSURE OPERATIONS AND GROUP ALGEBRAS
}

\author{
by J. D. P. MELDRUM AND D. A. R. WALLACE
}

(Received 22nd November 1971)

\section{Introduction}

Let $G$ be a group and let $K$ be a field. The twisted group algebra $K^{t}(G)$ of $G$ over $K$ is defined as follows: let $G$ have elements $a, b, c, \ldots$ and let $K^{t}(G)$ be the vector space over $K$ with basis elements $\bar{a}, \bar{b}, \bar{c}, \ldots$; let $\alpha: G \times G \rightarrow K$ be a 2-cocycle and define a multiplication on $K^{t}(G)$ by

$$
\bar{x} \bar{y}=\alpha(x, y) \overline{x y} \quad(x, y \in G)
$$

extending this by linearity to $K^{t}(G)$ yields an associative algebra. We are interested in information concerning the Jacobson radical of $K^{t}(G)$, denoted by $J K^{t}(G)$.

Extending an earlier notion ((14), p. 54 Definition) we call a class $X$ of groups a $J K^{t}$ class if whenever $G$ has a normal subgroup $H$ such that $G / H \in X$ then $J K^{t}(G) \subseteq J K^{t}(H) K^{t}(G)$. We call a class $X$ of groups an $N J K^{t}$ class of exponent $n>0$ if whenever $G$ has a normal subgroup $H$ such that $G / H \in X$ then $\left[J K^{t}(G)\right]^{n} \subseteq J K^{t}(H) K^{t}(G)$; we call $X$ an $N J K^{t}$ class if $J K^{t}(G)$ is nilpotent modulo $J K^{t}(H) K^{t}(G)$. If $K$ has characteristic 0 then the class of finite groups is a $J K^{t}$ class ((8) Proposition 1.5, p. 164). If $K$ has characteristic $p>0$ then the class of finite groups of order at most $n$ is an $N J K^{t}$ class of exponent $n$ ((8) Proposition 1.3 , (9) p. 164) and the class of finite soluble groups of orders $p^{a} m,(p, m)=1$, is an $N J K^{t}$ class of exponent $p^{a}((8)$ Theorem 1.6, (9) p. 164). We remark in passing that if a group $G$ belongs to an $N J K^{t}$ class of exponent $n$ then $\left[J K^{t}(G)\right]^{n}=\{0\}$; it is unknown whether the converse is true. In this paper we show that the property of being a $J K^{t}$ or an $N J K^{t}$ class is invariant under certain closure operations on classes of groups. This enables us to deduce that certain large classes of groups are of the above types and so have zero or nilpotent Jacobson radicals; further our methods provide means for extending results on $J K^{t}$ or $N J K^{t}$ classes.

Following the ideas of P. Hall (5) a mapping A from classes of groups to classes of groups is called a closure operation if, for classes $\boldsymbol{X}$ and $\boldsymbol{Y}$ of groups,

(i) $\boldsymbol{X} \subseteq \mathrm{A} \boldsymbol{X}$,

(ii) $\boldsymbol{Y} \subseteq \boldsymbol{X}$ implies $\mathrm{A} \boldsymbol{Y} \subseteq \mathrm{A} \boldsymbol{X}$ and

(iii) $A X=A A X$. 
$\boldsymbol{X}$ is A-closed if $\boldsymbol{X}=\mathrm{A} \boldsymbol{X}$. For every class $\boldsymbol{X}$ and closure operation A there is a least A-closed class containing $X$, namely $\mathrm{A} X$. We shall employ closure operations L, P and $\hat{\mathbf{P}}$ defined as follows ((5), p. 533):

$G \in \mathrm{L} X$ if every finitely generated subgroup of $G$ is contained in an $X$ subgroup of $G$;

$G \in \mathbf{P} X$ if there exists a series of subgroups $H_{i}$ of $G$ such that

$$
\{1\}=H_{0} \triangleleft H_{1} \triangleleft \ldots \triangleleft H_{n}=G
$$

where $H_{j+1} / H_{j} \in X(j=0,1, \ldots, n-1)$.

$G \in \hat{\mathbf{P}} \boldsymbol{X}$ if for some totally ordered set $\Omega$ there exists a family $\left\{U_{\sigma}, V_{\sigma}: \sigma \in \Omega\right\}$ of pairs of subgroups of $G$ such that

(i) $U_{\sigma} \triangleleft V_{\sigma}$ for all $\sigma \in \Omega$,

(ii) $V_{\sigma} / U_{\sigma} \in \boldsymbol{X}$ for all $\sigma \in \Omega$,

(iii) $\sigma<\tau(\sigma, \tau \in \Omega)$ implies that $V_{\sigma} \subseteq U_{\tau}$,

(iv) $G \backslash\{1\}=\bigcup_{\sigma \in \Omega}\left(V_{\sigma} \mid U_{\sigma}\right)$.

We shall show that $\mathbf{P}$ and $\hat{P}$ are closure operations.

Finally we use the standard notation that if $\mathrm{A}$ and $\mathrm{B}$ are two closure operations then $\{\mathrm{A}, \mathrm{B}\} \boldsymbol{X}$ denotes the least class of groups containing $\boldsymbol{X}$ and closed under $A$ and $B$.

Our main results are as follows.

Theorem 1.1. Let $X$ be a J $K^{t}$ class. Then $\{\mathrm{L}, \hat{\mathbf{P}}\} X$ is a $J K^{t}$ class.

Theorem 1.2. Let $X$ be an $N J K^{t}$ class. Then $P X$ is an NJK' class.

\section{Preliminary results}

In this section we collect together a few simpler results, some of which are necessary for later proofs and some of which are of independent interest. The following lemma, although well-known, is included for the sake of completeness.

Lemma 2.1. $\mathrm{P}$ and $\hat{\mathrm{P}}$ are closure operations.

Proof. First we show that $\mathbf{P}$ is a closure operation. The fact that $\mathbf{P}$ satisfies (i) and (ii) of the definition of a closure operation is immediate. Now let $G \in \mathbf{P P} X$. Then we have a series

$$
\{1\}=H_{0} \triangleleft H_{1} \triangleleft \ldots \triangleleft H_{n}=G
$$

such that $H_{i+1} / H_{i} \in \mathrm{P} X(i=0,1, \ldots, n-1)$. Thus we have for each $i$, $0 \leqq i \leqq n-1$, a series

$$
H_{i}=H_{i 0} \triangleleft H_{i 1} \triangleleft \ldots \triangleleft H_{i n_{i}}=H_{i+1}
$$

such that $H_{i j+1} / H_{i j} \in X\left(j=0,1, \ldots, n_{i}-1\right)$. Then the series

$$
\{1\}=H_{00} \triangleleft H_{01} \triangleleft \ldots \triangleleft H_{i j} \triangleleft H_{i j+1} \triangleleft \ldots \triangleleft H_{n}=G
$$


shows that $G \in \mathrm{P} X$. Hence $\mathrm{P}$ satisfies (iii) of the definition and is a closure operation.

To prove that $\boldsymbol{X} \subseteq \hat{\mathrm{P}} \boldsymbol{X}$ let $G \in X$ and consider the ordered set $\Omega=\{1\}$ and the pair $\{1, G\}$ of subgroups of $G$. The conditions set out in the definition of $\hat{\mathbf{P}} \boldsymbol{X}$ are then satisfied and so $G \in \hat{\mathrm{P} X}$. Thus $X \subseteq \hat{\mathbf{P}} X$. If $Y \subseteq X$ then it is immediate from the definition that $\hat{\mathbf{P}} \boldsymbol{Y} \subseteq \hat{\mathbf{P}} \boldsymbol{X}$.

It remains to show that $\hat{\mathbf{P}} \boldsymbol{X}=\hat{\mathbf{P}} \hat{\mathbf{P}} \boldsymbol{X}$. Let $G \in \hat{\mathbf{P}} \hat{\mathbf{P}} \boldsymbol{X}$. Then, for some totally ordered set $\Omega$, there exists a family $\left\{U_{\sigma}, V_{\sigma}: \sigma \in \Omega\right\}$ of pairs of subgroups such that

(i) $U_{\sigma} \triangleleft V_{\sigma}$ for all $\sigma \in \Omega$,

(ii) $V_{\sigma} / U_{\sigma} \in \hat{\mathbf{P}} \boldsymbol{X}$ for all $\sigma \in \Omega$,

(iii) $\sigma<\tau(\sigma, \tau \in \Omega)$, implies that $V_{\sigma} \subseteq U_{\tau}$

(iv) $G \backslash\{1\}=\bigcup_{\sigma \in \Omega}\left(V_{\sigma} \mid U_{\sigma}\right)$.

But (ii) implies that for each $\sigma \in \Omega$, there exists a totally ordered set $M_{\sigma}$ and a family $\left\{R_{\mu_{\sigma}}, S_{\mu_{\sigma}}: \mu_{\sigma} \in M_{\sigma}\right\}$ of pairs of subgroups such that

(i) $R_{\mu_{\sigma}} \triangleleft S_{\mu_{\sigma}}$ for all $\mu_{\sigma} \in M_{\sigma}$,

(ii) $S_{\mu_{\sigma}} / R_{\mu_{\sigma}} \in X$ for all $\mu_{\sigma} \in M_{\sigma}$,

(iii) $\mu_{\sigma}<v_{\sigma}\left(\mu_{\sigma}, v_{\sigma} \in \Omega_{\sigma}\right)$ implies that $S_{\mu_{\sigma}} \subseteq R_{v_{\sigma}}$,

(iv) $V_{\sigma} \mid U_{\sigma}=\bigcup_{\mu_{\sigma} \in M_{\sigma}}\left(S_{\mu_{\sigma}} \mid R_{\mu_{\sigma}}\right)$.

Let $\bar{\Omega}=\bigcup_{\sigma \in \Omega} M_{\sigma}$ be totally ordered by the relation $\mu_{\sigma}<v_{\tau}$ if either $\sigma<\tau$ or $\sigma=\tau$ and $\mu_{\sigma}<v_{\sigma}$. Then the family $\left\{R_{\mu_{\sigma}}, S_{\mu_{\sigma}}: \mu_{\sigma} \in \bar{\Omega}\right\}$ of pairs of subgroups satisfies

(i) $R_{\mu_{\sigma}} \triangleleft S_{\mu_{\sigma}}$ for all $\mu_{\sigma} \in \bar{\Omega}$,

(ii) $S_{\mu_{\sigma}} / R_{\mu_{\sigma}} \in X$ for all $\mu_{\sigma} \in \bar{\Omega}$,

(iii) $\mu_{\sigma}<v_{\tau}\left(\mu_{\sigma}, v_{\tau} \in \bar{\Omega}\right)$ implies that $S_{\mu_{\sigma}} \subseteq R_{v_{\tau}}$ if $\sigma=\tau$ and that

$$
S_{\mu_{\sigma}} \subseteq V_{\sigma} \subseteq U_{\tau} \subseteq R_{v_{\tau}} \text { if } \sigma<\tau,
$$

(iv) $G \backslash\{1\}=\bigcup_{\mu_{0} \in \overline{\mathbf{\Omega}}}\left(S_{\mu_{\sigma}} \mid R_{\mu_{\sigma}}\right)$.

Thus $G \in \hat{\mathbf{P}} \boldsymbol{X}$ and so $\hat{\mathbf{P}} \hat{\mathbf{P}} \boldsymbol{X}=\hat{\mathbf{P}} \boldsymbol{X}$. Consequently $\hat{\mathbf{P}}$ is a closure operation.

Lemma 2.2. Let $X$ be a $J K^{t}$ class. Then $\mathrm{L} X$ is a $J K^{t}$ class.

Proof. Let $G$ be a group, let $H \triangleleft G$ and suppose $G / H \in \mathrm{L} X$. We wish to show that $J K^{t}(G) \subseteq J K^{t}(H) K^{t}(G)$. Let $f \in J K^{\prime}(G)$, then $f=\sum_{i=1}^{n} \lambda_{i} \bar{g}_{i}$ where $\lambda_{j} \in K, g_{j} \in G(j=1,2, \ldots, n)$. Let $R$ be the subgroup of $G$ generated by 
$H \cup\left\{g_{1}, g_{2}, \ldots, g_{n}\right\}$. Then since $G / H \in \mathrm{L} X$ and $R / H$ is finitely generated there exists a subgroup $S$ of $G$ such that $R \subseteq S$ and $S / H \in X$. But $f \in J K^{t}(G) \cap K^{t}(S)$ and so $f \in J K^{t}(S)((8)$ Lemma 1.9, (9) p. 164). Since $S / H \in X$ we have

$$
f \in J K^{t}(S) \subseteq J K^{t}(H) K^{t}(S) \subseteq J K^{t}(H) K^{t}(G)
$$

which establishes the lemma.

Theorem 2.3. Let $X$ be an $N J K^{t}$ class and let $G$ be a group and $H$ a normal subgroup of $G$ such that $G / H \in \mathrm{L} X$. Then $J K^{t}(G)$ is locally nilpotent modulo $J K^{t}(H) K^{t}(G)$.

Proof. Let $f_{1}, f_{2}, \ldots, f_{r}$ be $r$ elements of $J K^{t}(G)$. We require to show that the ring $W$ generated by $\left\{f_{1}, f_{2}, \ldots, f_{r}\right\}$ is nilpotent modulo $J K^{\prime}(H) K^{\prime}(G)$. In the expressions for the $f_{i}$ as linear combinations of elements of $G$ let $F$ be the finite set of elements of $G$ appearing with non-zero coefficients. Let $R$ be the subgroup generated by $H \cup F$. Then since $G / H \in \mathrm{L} X$ and $R / H$ is finitely generated there exists a subgroup $S$ of $G$ such that $R \subseteq S$ and $S / H \in X$. But $f_{i} \in J K^{t}(G) \cap K^{t}(S)$ and so $f_{i} \in J K^{t}(S)(i=1,2, \ldots, r)((8)$ Lemma 1.9); thus $W \subseteq J K^{t}(S)$. But $X$ is an $N J K^{t}$ class and hence there exists $n$ such that

$$
\left[J K^{t}(S)\right]^{n} \subseteq J K^{t}(H) K^{t}(S) \subseteq J K^{t}(H) K^{t}(G) .
$$

This implies that $W^{n} \subseteq J K^{t}(H) K^{t}(G)$ which completes the proof.

\section{Proofs of main theorems}

Theorem 1.1 follows directly from Lemma 2.2 and the next result.

Lemma 3.1. Let $\boldsymbol{X}$ be a $J K^{t}$ class. Then $\hat{\mathbf{P}} \boldsymbol{X}$ is a $J K^{t}$ class.

Proof. Let $G$ have a normal subgroup $H$ such that $G / H \in \hat{\mathbf{P} X}$. Let the series satisfying the definition of the closure operation be given by the family $\left\{V_{\sigma} / H, U_{\sigma} / H: \sigma \in \Omega\right\}$, where $\Omega$ is a totally ordered set, $U_{\sigma} \sqsupset V_{\sigma}, V_{\sigma} / U_{\sigma} \in X$ and $G \backslash H=\bigcup_{\sigma \in \Omega}\left(V_{\sigma} \mid U_{\sigma}\right)$. Let $T$ be a fixed transversal of $H \operatorname{in} G$. Consider $f \in J K^{t}(G)$, then $f=\sum_{i=1}^{n} \lambda_{i} \bar{g}_{i}$ where $\lambda_{j} \in K^{t}(H), g_{j} \in T(j=1,2, \ldots, n)$. We wish to show that $\lambda_{j} \in J K^{t}(H)(j=1,2, \ldots, n)$ and we shall do this by induction on $n=l(f)$, the coset length of $f$. If $g_{j} \in V_{\sigma(j)} \mid U_{\sigma(j)}(j=1,2, \ldots, n)$ then we may first suppose $f \in J K^{t}\left(V_{\tau}\right)$ where $\tau=\max \{\sigma(1), \sigma(2), \ldots, \sigma(n)\}$ since clearly $f \in K^{t}\left(V_{\tau}\right)$ and so $f \in J K^{t}(G) \cap K^{t}\left(V_{\tau}\right) \subseteq J K^{t}\left(V_{\tau}\right)((8)$, Lemma 1.9). We shall argue, by induction on this coset length, that if $f \in J K^{t}\left(V_{\rho}\right)$ for some $\rho$ then

$$
f \in J K^{t}(H) K^{\prime}(G) \text {. }
$$

We begin with the observation that $\bar{g}_{i}(i=1,2, \ldots, n)$ is invertible and that $\bar{g}_{i}^{-1}=c_{i}{\overline{g_{i}^{-1}}}^{-1}$ where $c_{i} \in K, c_{i} \neq 0(i=1,2, \ldots, n)((\mathbf{8})$, Lemma 1.1).

Suppose $n=1$ and then $f=\lambda_{1} \bar{g}_{1}$. As $J K^{t}\left(V_{\tau}\right)$ is an ideal of $K^{t}\left(V_{t}\right)$ we have $\lambda_{1}=f \bar{g}_{1}^{-1} \in J K^{t}\left(V_{\tau}\right)$ and hence $\lambda_{1} \in J K^{t}\left(V_{\tau}\right) \cap K^{t}(H) \subseteq J K^{t}(H)((8)$, Lemma 1.9). Thus $f \in J K^{t}(H) K^{t}\left(V_{\tau}\right)$. 
Suppose now $n>1$ and assume the result is true for those $f$ with $l(f)<n$. Then $f \bar{g}_{1}^{-1} \in J K^{t}\left(V_{\tau}\right)$ and

$$
\begin{aligned}
f \bar{g}_{1}^{-1} & =\sum_{i=1}^{n} \lambda_{i} \bar{g}_{i} \bar{g}_{1}^{-1}=\sum_{i=1}^{n} \lambda_{i} \bar{g}_{i} c_{1} \overline{g_{1}^{-1}} \\
& =\sum_{i=1}^{n} \lambda_{i} c_{1} \alpha\left(g_{i}, g_{1}^{-1}\right) \overline{g_{i} g_{1}^{-1}}
\end{aligned}
$$

In terms of the new transversal $T g_{1}^{-1}=\left\{\operatorname{tg}_{1}^{-1}: t \in T\right\}$ we are required to show that $\lambda_{j} c_{1} \alpha\left(g_{j}, g_{1}^{-1}\right) \in J K^{t}(H)$, from which we deduce easily that $\lambda_{j} \in J K^{t}(H)$ $(j=1,2, \ldots, n)$. Consequently, without loss of generality, we may assume $f$ has the form $f=\sum_{i=1}^{n} \lambda_{i} \bar{g}_{i}$ where $g_{1}=1$. The set $\left\{g_{1}, g_{2}, \ldots, g_{n}\right\}$ is not necessarily a subset of a transversal of $U_{\tau}$ in $V_{\tau}$ and accordingly it is convenient to make a notational change. Let

$$
\left\{g_{1}, g_{2}, \ldots, g_{n}\right\}=\left\{g_{11}, g_{12}, \ldots, g_{1 d_{1}} ; g_{21}, g_{22}, \ldots, g_{2 d_{2}} ; \ldots ; g_{s 1}, g_{s 2}, \ldots, g_{s d_{s}}\right\}
$$

where $d_{1}+d_{2}+\ldots+d_{s}=n$ and $U_{\tau} g_{i j}=U_{\tau} g_{k l}$ if and only if

$$
i=k\left(j=1,2, \ldots, d_{i} ; l=1,2, \ldots, d_{k} ; i, k=1,2, \ldots, s\right)
$$

and let $f=\sum_{i=1}^{n} \lambda_{i} \bar{g}_{i}=\sum_{j=1}^{s} \sum_{k=1}^{d j} \mu_{j k} \bar{g}_{j k}$ where $\mu_{j k}=\lambda_{i}$ for some $i=i(j, k)$. Since $g_{j} \in V_{\tau} / U_{\tau}$ for some $j \neq 1$ and $g_{1} \in H$ we know that in the above we have $s>1$ and hence $d_{i}<n(i=1,2, \ldots, s)$. Let $g_{i j}=u_{i j} g_{i 1}$ where $u_{i j} \in U_{\tau}$ $\left(j=1,2, \ldots, d_{i} ; i=1,2, \ldots, s\right)$. Then

$$
\begin{aligned}
f & =\sum_{j=1}^{s} \sum_{k=1}^{d i} \mu_{j k} \bar{g}_{j k} \\
& =\sum_{j=1}^{s} \sum_{k=1}^{d j} \mu_{j k} \overline{u_{j k} g_{j 1}} \\
& =\sum_{j=1}^{s} \sum_{k=1}^{d j} \mu_{j k} \alpha\left(u_{j k}, g_{j 1}\right) \bar{u}_{j k} \bar{g}_{j 1} \\
& =\sum_{j=1}^{s}\left[\sum_{k=1}^{d j} \mu_{j k} \alpha\left(u_{j k}, g_{j 1}\right) \bar{u}_{j k}\right] \bar{g}_{j 1} .
\end{aligned}
$$

Since $V_{\mathfrak{t}} / U_{\tau} \in X$ we deduce that

$$
\sum_{k=1}^{d j} \mu_{j k} \alpha\left(u_{j k}, g_{j 1}\right) \bar{u}_{j k} \in J K^{\prime}\left(U_{\imath}\right) \quad(j=1,2, \ldots, s) .
$$

Since $\Omega$ is totally ordered there exists $\sigma \leqq \tau$ such that $u_{i j} \in V_{\sigma}\left(j=1,2, \ldots, d_{i}\right.$; $i=1,2, \ldots, s)$ and hence

$$
\sum_{k=1}^{d j} \mu_{j k} \alpha\left(u_{j k}, g_{j 1}\right) \bar{u}_{j k} \in K^{\prime}\left(V_{a}\right) \quad(j=1,2, \ldots, s)
$$


which implies that

But

$$
\sum_{k=1}^{d j} \mu_{j k} \alpha\left(u_{j k}, g_{j 1}\right) \bar{u}_{j k} \in J K^{\prime}\left(V_{\sigma}\right) \quad(j=1,2, \ldots, s) .
$$

and so, by our induction hypothesis,

$$
l\left(\sum_{k=1}^{d j} \mu_{j k} \alpha\left(u_{j k}, g_{j 1}\right) \bar{u}_{j k}\right) \leqq d_{j}<n \quad(j=1,2, \ldots, s)
$$

$$
\sum_{k=1}^{d j} \mu_{j k} \alpha\left(u_{j k}, g_{j 1}\right) \bar{u}_{j k} \in J K^{t}(H) K^{\prime}(G) \quad(j=1,2, \ldots, s) .
$$

But $J K^{t}(H) K^{t}(G)$ is an ideal of $K^{t}(G)((12)$, Theorem 4.1), and so

$$
f \in J K^{t}(H) K^{\prime}(G) \text {. }
$$

This completes the induction and establishes the lemma.

Proof of Theorem 1.2. Let $G$ have a normal subgroup $H$ such that $G / H \in \mathrm{P} X$. Then there exists a series

$$
H=H_{0} \triangleleft H_{1} \triangleleft \ldots \triangleleft H_{n}=G
$$

such that $H_{i+1} / H_{i} \in X(i=0,1, \ldots, n-1)$. Since $H_{n} / H_{n-1} \in X$ there exists $s>0$ such that $\left[J K^{t}\left(H_{n}\right)\right]^{s} \subseteq J K^{t}\left(H_{n-1}\right) K^{t}\left(H_{n}\right)$. We argue by induction on $n$ and suppose that we have shown that there exists $r_{n-1}>0$ such that

$$
\left[J K^{t}\left(H_{n-1}\right)\right]^{r_{n-1}} \subseteq J K^{t}(H) K^{t}\left(H_{n-1}\right) .
$$

We also have, since $H_{n-1} \triangleleft H_{n}$, that $J K^{t}\left(H_{n-1}\right) K^{t}\left(H_{n}\right)=K^{t}\left(H_{n}\right) J K^{t}\left(H_{n-1}\right)$ ((8), lemma 1.2). Hence we deduce that

$$
\begin{aligned}
{\left[J K^{t}\left(H_{n}\right)\right]^{r_{n-1}} } & \subseteq\left[J K^{t}\left(H_{n-1}\right) K^{t}\left(H_{n}\right)\right]^{r_{n-1}} \\
& \subseteq\left[J K^{t}\left(H_{n-1}\right)\right]^{r_{n-1}} K^{t}\left(H_{n}\right) \\
& \subseteq J K^{t}(H) K^{t}\left(H_{n-1}\right) K^{t}\left(H_{n}\right) \\
& \subseteq J K^{t}(H) K^{t}\left(H_{n}\right) .
\end{aligned}
$$

The step $n=1$ is trivial. This establishes the induction and so we conclude that

$$
\begin{aligned}
{\left[J K^{t}(G)\right]^{r_{n}} } & =\left[J K^{t}\left(H_{n}\right)\right]^{r_{n}} \\
& \subseteq J K^{t}(H) K^{t}\left(H_{n}\right)=J K^{t}(H) K^{t}(G) .
\end{aligned}
$$

This establishes Theorem 1.2.

\section{Concluding remarks}

Our results on semisimplicity etc. are applicable to large classes of groups. Some of these applications are incorporated in the next two theorems whose corollaries contain some known results ((2), (3), (4), (9), (10), (12), (14), (15)). 
Theorem 4.1. Let $K$ have characteristic 0 and let $X_{0}$ be the class of finite groups. Then $\{\mathrm{L}, \hat{\mathrm{P}}\} X_{0}$ is a $J K^{t}$ class.

Corollary. Let $K$ have characteristic 0 and let $G$ be one of the following types:

(a) locally finite group,

(b) locally soluble group,

(c) $S N$ group,

(d) residually finite group,

(e) free group.

Then $J K^{\prime}(G)=0$.

Theorem 4.2. Let $K$ have characteristic $p>0$ and let $X_{p}$ be the class of finite groups of orders prime to $p$. Then $\{\mathrm{L}, \hat{\mathbf{P}}\} X_{p}$ is a $J K^{t}$ class.

Corollary. Let $K$ have characteristic $p>0$ and let $G$ be one of the following types:

(a) locally finite group with no non-trivial p-elements,

(b) locally soluble group, the factors of the derived series of the finitely generated subgroups of $G$ having no non-trivial p-elements,

(c) SN group, the factors of an abelian series having no non-trivial p-elements,

(d) residually finite (of order prime to $p$ ) group,

(e) free group.

Then $J K^{t}(G)=\{0\}$.

In order to obtain further results in the above manner we would remark that it is possible to extend Theorem 1.2 if we allow well-ordered series and if we are willing to be satisfied with local nilpotency. We define, therefore, an operation $\overline{\text { PI }}$ as follows (cf. (6), p. 171 and p. 182):

$G \in \overline{\mathrm{PI}} X$ if there exists an ordinal $\sigma$ and a series $\left\{H_{\mu}: \mu \leqq \sigma\right\}$ of subgroups such that

(i) $H_{\lambda} \triangleleft G(\lambda \leqq \sigma)$,

(ii) if $\lambda$ is a limit ordinal then $H_{\lambda}=\bigcup_{\mu<\lambda} H_{\mu} \quad(\lambda \leqq \sigma)$,

(iii) $H_{\lambda+1} / H_{\lambda} \in X(\lambda<\sigma)$ and

(iv) $1=H_{0}, H_{\sigma}=G$.

If in the above (i) is replaced by

(i) $H_{\lambda} \triangleleft H_{\lambda+1}(\lambda<\sigma)$,

then we say $G \in \dot{P} X$.

$\overline{\text { PI }}$ satisfies (i) and (ii) of the definition of a closure operation but not (iii). The subsequent result is unfortunately restricted to the operation $\overline{\mathbf{P I}}$ and cannot be extended to the least closure operation containing $\overline{\mathbf{P I}}$. 
Theorem 4.3. Let $X$ be an NJK' class. Let $G$ be a group and let $H$ be $a$ normal subgroup of $G$ such that $G / H \in \overline{\mathrm{PI}} X$. Then $J K^{t}(G)$ is locally nilpotent modulo $J K^{t}(H) K^{t}(G)$.

Proof. By assumption there exists an ordinal $\sigma$ and a series

$$
H=H_{0} \subseteq H_{1} \subseteq \ldots \subseteq H_{\sigma}=G
$$

with $H_{\alpha} \triangleleft G, H_{\lambda+1} / H_{\lambda} \in X$ and if $\lambda$ is a limit ordinal $H_{\lambda}=\bigcup_{\alpha<\lambda} H_{\alpha}$. We shall establish the theorem by showing that any finitely generated subring of $J K^{t}\left(H_{\alpha}\right) K^{t}(G)$ is nilpotent modulo $J K^{t}(H) K^{t}(G)$ for all $\alpha \leqq \sigma$. If this statement is not true then there exists a least ordinal $\beta$ (say) such that $J K^{t}\left(H_{\beta}\right) K^{t}(G)$ contains a finitely generated subring $R$ which is not nilpotent modulo

$$
J K^{t}(H) K^{t}(G) \text {. }
$$

Let $R$ be generated by $f_{1}, f_{2}, \ldots, f_{n}$. If $\beta$ is a limit ordinal then $H_{\beta}=\bigcup_{\alpha<\beta} H_{\alpha}$ and we may write

$$
f_{i}=x_{i 1} \bar{g}_{1}+x_{i 2} \bar{g}_{2}+\ldots+x_{i r} \bar{g}_{r}
$$

where $\left\{g_{1}, g_{2}, \ldots, g_{r}\right\}$ is part of a transversal of $H_{\beta}$ in $G$ and $x_{i j} \in J K^{t}\left(H_{\beta}\right)$ $(j=1,2, \ldots, r ; i=1,2, \ldots, n)$. Since $x_{i j}$ is either 0 or has finite support there exists $\delta<\beta$ such that, for all $i, j, x_{i j} \in K^{t}\left(H_{\delta}\right)$. Then, as $H_{\delta} \subseteq H_{\beta}$,

$$
x_{i j} \in J K^{t}\left(H_{\beta}\right) \cap K^{t}\left(H_{\delta}\right) \subseteq J K^{t}\left(H_{\delta}\right) \quad((8), \text { Lemma 1.9). }
$$

Thus $f_{i} \in J K^{t}\left(H_{\delta}\right) K^{t}(G) \quad(i=1,2, \ldots, r)$ and so $R \subseteq J K^{t}\left(H_{\delta}\right) K^{t}(G)$. This contradicts the definition of $\beta$ and hence $\beta$ cannot be a limit ordinal. Thus let $\beta=\lambda+1$ and then we have $H_{\beta} / H_{\gamma} \in X$. Since $\boldsymbol{X}$ is an $N J K^{t}$ class there exists an integer $m$ such that $\left[J K^{t}\left(H_{\beta}\right)\right]^{m} \subseteq J K^{t}\left(H_{\gamma}\right) K^{t}\left(H_{\beta}\right)$. Hence, as $H_{\beta} \triangleleft G$, we have ((8), Lemma 1.2, (12) Theorem 4.1)

$$
\begin{aligned}
R^{m} & \subseteq\left[J K^{t}\left(H_{\beta}\right) K^{t}(G)\right]^{m} \\
& =\left[J K^{t}\left(H_{\beta}\right)\right]^{m} K^{t}(G) \\
& \subseteq J K^{t}\left(H_{\gamma}\right) K^{t}\left(H_{\beta}\right) K^{t}(G) \\
& =J K^{t}\left(H_{\gamma}\right) K^{t}(G) .
\end{aligned}
$$

But $R^{m}$ is a finitely generated subring of $J K^{t}\left(H_{\gamma}\right) K^{t}(G)$ and, by the assumption on $\beta$, is nilpotent modulo $J K^{t}(H) K^{t}(G)$; this is a contradiction to the choice of $R$. Hence any finitely generated subring of $J K^{t}\left(H_{a}\right) K^{t}(G)$ is nilpotent modulo $J K^{t}(H) K^{t}(G)$ for all $\alpha \leqq \sigma$ and by taking $\alpha=\sigma$ we obtain the desired result.

Lemma 4.4. Let $G$ be a group and let $H$ be a normal subgroup such that $G / H$ is abelian or locally finite. Then $J K^{t}(G)$ is locally nilpotent modulo $J K^{t}(H) K^{t}(G)$.

Proof. By Theorem 2.4 if $G / H$ is abelian then the result follows from Lemma 3.2 of (15) and if $G / H$ is locally finite the result follows from Proposition 1.3 of (8). 
From this Lemma we deduce two useful results. For convenience we let $\boldsymbol{F}$ be the class of finite groups and let $S$ be the class of soluble groups.

Theorem 4.5. Let $G$ be a group and let $H$ be a normal subgroup such that $G / H \in \mathrm{L}(F \cup S)$. Let $J K^{t}(H)=\{0\}$ and if $K$ has characteristic $p>0$ let $G$ have no non-trivial p-elements. Then $J K^{t}(G)=\{0\}$.

Proof. We argue by contradiction and so we suppose that $x \in J K^{t}(G)$, $x \neq 0$. Let $G_{0}$ be the group generated by $H$ and by the support of $x$. Then $G_{0} / H$ is finitely generated and so is either finite or soluble. If $G_{0} / H$ is finite then, by Lemma $4.4, J K^{t}\left(G_{0}\right)$ is locally nilpotent. If $K$ has characteristic 0 then finite groups form a $J K^{t}$ class and $J K^{t}\left(G_{0}\right)=\{0\}$. If $K$ has characteristic $p>0$, by Theorem 3.2 of $(8)$, we have $J K^{\prime}\left(G_{0}\right)=\{0\}$.

Suppose therefore $G_{0} / H$ is soluble. Then there exists a series

$$
G_{0} \triangleright G_{1} \triangleright \ldots \triangleright G_{m}=H
$$

such that $G_{i} \triangleleft G$ and $G_{i} / G_{i+1}$ is abelian $(i=0,1, \ldots, m-1)$. If $m=1$ then, again by Lemma 4.4,JKt $\left(G_{0}\right)$ is locally nilpotent. If $K$ has characteristic 0 this implies $J K^{t}\left(G_{0}\right)=\{0\}$ (cf. (7), (9), (15), Lemma 3.3), and if $K$ has characteristic $p>0$, since $G$ has no non-trivial $p$-elements, this implies $J K^{t}\left(G_{0}\right)=\{0\} \quad((\boldsymbol{8})$ Theorem 3.2). Suppose now $m>1$. We argue by induction and suppose the result is true for $m-1$. Thus we suppose $J K^{t}\left(G_{1}\right)=\{0\}$. By the above proof we have $J K^{t}\left(G_{0}\right)=\{0\}$, establishing the theorem.

Finally we apply this result to obtain the following (cf. (15), Theorem 3.5).

Theorem 4.6. Let $G \in \mathrm{P} L(F \cup S)$. If $K$ has characteristic 0 or if $K$ has characteristic $p>0$ and $G$ has no non-trivial p-elements then $J K^{t}(G)=\{0\}$.

Proof. By assumption $G$ has a series $\left\{H_{\lambda}: \lambda \leqq \sigma\right\}$ of subgroups satisfying the definition of $\operatorname{PL}(F \cup S)$. Suppose $J K^{t}(G) \neq\{0\}$ and let $\beta$ be the least ordinal such that $J K^{t}\left(H_{\beta}\right) \neq\{0\}$. By assumption $J K^{t}\left(H_{\alpha}\right)=0(\alpha<\beta)$. Let $f \in J K^{t}\left(H_{\beta}\right)$, $f \neq 0$. If $\beta$ is a limit ordinal then, as $f$ has finite support, there exists $\delta<\beta$ such that $f \in K^{t}\left(H_{\delta}\right)$. Thus, as $H_{\delta} \subseteq H_{\beta}$ we have $f \in J K^{t}\left(H_{\beta}\right) \cap K^{t}\left(H_{\delta}\right) \subseteq J K^{t}\left(H_{\delta}\right)$ which contradicts the choice of $\beta$. Hence $\beta$ is not a limit ordinal and thus we have $\beta=\lambda+1$. Then, by hypothesis, $J K^{t}\left(H_{\gamma}\right)=\{0\}$. Since

$$
H_{\gamma+1} / H_{\gamma} \in \mathrm{L}(\boldsymbol{F} \cup S)
$$

we deduce from Theorem 4.4 that $J K^{t}\left(H_{\gamma+1}\right)=\{0\}$ and this contradicts the choice of $\beta$. Thus the theorem is proved.

\section{REFERENCES}

(1) A. A. BovDI, Group rings of torsion-free groups, Sibirsk. Math. Z. 1 (1960), 251-253.

(2) I. G. ConNell, On the group ring, Canad. J. Math. 15 (1963), 650-685. 
(3) J. A. Green and S. E. Stonehewer, The radicals of some group algebras, J. Algebra 13 (1969), 137-142.

(4) J. A. Green and S. E. Stonehewer, Note on the paper " The radicals of some group algebras ", J. Algebra 13 (1969), 297.

(5) P. Hall, On non-strictly simple groups, Proc. Cambridge Philos. Soc. 59 (1963), 531-553.

(6) A. G. KuROSH, Theory of Groups, vol. II (New York, 1956).

(7) D. S. Passman, Nil ideals in group rings, Michigan Math. J. 9 (1962), 375-384.

(8) D. S. Passman, Radicals of twisted group rings, Proc. London Math. Soc. (3) 20 (1970), 409-437.

(9) D. S. Passman, On the semisimplicity of twisted group algebras, Proc. Amer. Math. Soc. 25 (1970), 161-166.

(10) S. E. Stonehewer, Group algebras of some torsion-free groups, J. Algebra 13 (1969), 143-147.

(11) O. E. Villamayor, On the semisimplicity of group algebras, Proc. Amer. Math. Soc. 9 (1958), 621-627.

(12) O. E. Villamayor, On the semisimplicity of group algebras II, Proc. Amer. Math. Soc. 10 (1959), 27-31.

(13) D. A. R. Wallace, The Jacobson radicals of the group algebras of a group and of certain normal subgroups, Math. Z. 100 (1967), 282-294.

(14) D. A. R. WAllace, Some applications of subnormality in groups in the study of group algebras, Math. Z. 108 (1968), 53-62.

(15) D. A. R. WALLACE, The radical of the group algebra of a subgroup, of a polycyclic group and of a restricted SN-group, Proc. Edinburgh Math. Soc. 17 (1970), 165-171.

UNIVERSITY OF EDINBURGH

UNIVERSITY OF ABERDEEN 